'A Riedel, 'M Bohnert, 'C Scholz, 'T Laengin, 'W Melchior, 'E Elke, '2M Gencay. 'Roche Diagnostics GmbH, Penzberg, Germany; ${ }^{2}$ Roche Diagnostics International, Rotkreuz, Switzerland

Background HSV infections during pregnancy are generally asymptomatic and associated with high rate of neonatal morbidity and death. Differential serologic diagnosis of HSV infections in pregnancy is important for the correct assessment of the infection status. The aim of this study was to evaluate the performance of Roche Elecsys ${ }^{\circledR}$ assays for the type specific determination of HSV-1 and HSV-2 IgG antibodies.

Methods A total of 800 samples obtained from sexually active adults $(n=300)$, pregnant women $(n=400)$ and herpes infection suspected patients $(n=100)$ were analysed using Roche Elecsys ${ }^{\circledR}$ HSV-1 and HSV-2 assays and commercially available Focus Diagnostics HerpeSelect, Radim HERPES S.V and Diasorin LIAISON type specific ELISA assays.

A commercially available Western Blot assay was used for the resolution of the discrepant results.

For further confirmation of the type specific detection performance of the Elecsys ${ }^{\circledR}$ HSV-1 and HSV-2 assays 310 potentially cross reactive samples for HSV-2 and 123 samples for HSV-1 were analysed.

Results The Elecsys ${ }^{\circledR}$ HSV-1 IgG assay showed a relative sensitivity of 95.6 to $100 \%$. The relative specificity was between 97.6 and $100 \%$. The Elecsys ${ }^{\circledR}$ HSV-2 IgG assay showed a relative sensitivity of 92.6 to $100 \%$. The relative specificity was between 98.7 and $100 \%$. These assays exhibits excellent differentiation between HSV-1 and HSV-2 infections with no cross reactivity to other herpes viruses like CMV, VZV or EBV.

Conclusion The results obtained with the type specific Roche Elecsys ${ }^{\circledR}$ HSV-1 and HSV-2 assays indicate that these assays are useful, specific and sensitive for the differential determination of HSV-1 and HSV-2 IgG antibodies in serum or plasma samples. The advantage of the Roche Elecsys ${ }^{\circledR}$ assays compared to ELISA based assays, is that these assays are rapid and can be performed in a fully automated process.

\section{P5.072 THE PERFORMANCE OF TWO IGG ELISA METHODS TO DETECT HSV-2 INFECTIONS AMONG SOUTH-AFRICAN WOMEN WHO ARE AT HIGHER RISK OF BECOMING HIV INFECTED}

doi:10.1136/sextrans-2013-051184.1116

II De Baetselier, 'E Rammutla, ${ }^{2} \mathrm{~K}$ Ahmed, ${ }^{3} \mathrm{~J}$ Deese, ${ }^{3} \mathrm{~L}$ Van Damme, ' $\mathbf{T}$ Crucitti. 'Institute of Tropical Medicine, Antwerp, Belgium; ${ }^{2}$ Setshaba Research Center, Soshanguve, Pretoria, South Africa; ${ }^{3}$ FHI-360, Durham, NC, United States

Background Specimens collected in Pretoria for the FEM-PrEP study, a phase III trial on pre-exposure prophylaxis for HIV prevention among African women, were tested for Herpes Simplex Virus type II (HSV-2). We present here the performance of the Focus HerpeSelect and Kalon HSV-2 gG2 ELISA.

Methods The HSV-2 infection was determined in 701 women at baseline with two IgG ELISAs: Kalon HSV-2 gG2 ELISA (Kalon Biologicals Ltd.) and HerpeSelect HSV IgG ELISA (Focus Technologies). Participants were considered true positive for HSV-2 when specimens were reactive in both assays. In order to determine incident HSV-2 infections during the study, specimens collected at final visit (i.e. after 52 weeks/at product interruption visit) of participants being HSV-2 seronegative at baseline were tested using the same assays.

Results At baseline, 287 and 315 positive results were found using the Kalon- and Focus assay, respectively. All Kalon positives were also positive in the Focus assay and considered to be true infections (initial prevalence: $40.9 \%$ ). Of the 28 specimens positive with the Focus only, 10 of them became true positive at final visit. We therefore assume that the Kalon missed 10 infections and, the Focus detected falsely 18 positives at baseline, resulting in a final HSV-2 prevalence of $42.4 \%$ at baseline. At final visit, an additional 33 new infections were found. At baseline we obtained a sensitivity of 100\% (95\% CI: 98.8-100) and 96.6\% (95\% CI: 93.9-98.4) and a specificity of $95.5 \%$ (95\% CI: 93.1-97.3) and 100\% (95\% CI: 99.1-100) for Focus and Kalon respectively.

Conclusion Although our study confirms the assay performance findings of previous studies in Sub-Saharan countries, we found less pronounced differences in terms of sensitivity and specificity of both assays using the cut-off as prescribed by the manufacturers. The prevalence of HSV-2 found in our study corresponds to previously reported results.

\section{P5.073 COMPARISON OF COBAS $\circledast 4800$ HPV ASSAY TO DIGENE HYBRID CAPTURE 2, ROCHE LINEAR ARRAY, AND AMPLICOR IN THE DETECTION OF HIGH-RISK HUMAN PAPILLOMAVIRUS GENOTYPES IN WOMEN WITH PREVIOUS ABNORMAL PAP SMEARS}

doi:10.1136/sextrans-2013-051184.1117

1,2,3S N Tabrizi, 'S Phillips, 1,2,3S M Garland. 'Regional HPV Reference Laboratory, Department of Microbiology and Infectious Diseases, The Royal Women's Hospital, Parkville, Australia; ${ }^{2}$ Department of Obstetrics and Gynaecology, University of Melbourne, Parkville, Australia; ${ }^{3}$ Murdoch Childrens Research Institute, Parkville, Australia

Introduction Cobas ${ }^{\circledR} 4800 \mathrm{HPV}$ assay has been evaluated as a screening and triage application recently. The aim of this study was to evaluate the performance of the Cobas ${ }^{\circledR} 4800 \mathrm{HPV}$ assay for the detection and identification of high-risk (HR) HPV genotypes after treatment of high grade lesion by comparison with the Hybrid Capture $2^{\circledR}$ (HC2), Amplicor (Amp), and Linear Array (LA) HPV tests. Methods Four hundred and six PreservCyt ${ }^{\circledR}$ specimens from women undergoing management for a high-grade Pap abnormality were evaluated and results compared with the HC2, Amp, and LA HPV test results, in addition to histological diagnosis of a contemporaneously collected biopsy.

Results The sensitivity in detection of underlying high-grade histological diagnosis by Cobas ${ }^{\circledR} 4800$ HPV was $90.6 \%$, for HC2 $86.1 \%$, whilst for Amp and LA 92.9\% and 91.8\% respectively. Restricting detection of Cobas ${ }^{\circledR} 4800 \mathrm{HPV}$ to only types 16 and 18 resulted in sensitivity of $60.0 \%$. Detection of HR genotypes by Cobas ${ }^{\circledR} 4800$ HPV showed a concordance of $86.9 \%, 96.1 \%$, and $96.3 \%$ when compared to HC2, Amp and LA respectively. Detection of HPV 16 and 18 by Cobas $^{\circledast} 4800$ HPV showed a concordance of $97.3 \%$ and $99 \%$ respectively when compared to LA.

Conclusion The performance of Cobas ${ }^{\circledR} 4800 \mathrm{HPV}$ was equivalent to the Amp and LA HPV tests for HR HPV detection. Cobas ${ }^{\circledR} 4800$ HPV identified more underlying histologically-confirmed high-grade lesions than the HC2 HPV test, with the added advantage of identifying HPV 16 and 18 genotypes present.

\section{P5.074 IMMUNE ACTIVATION AFTER STIMULATION WITH CRYPTOCOCCUS NEOFORMANS ANTIGENS PRE AND POST ART INITIATION IN HIV-1 POSITIVE UGANDANS}

doi:10.1136/sextrans-2013-051184.1118

'R N Sanya, ${ }^{2 D}$ Chatterjea, ${ }^{3} \mathrm{~A}$ Akampurrira, ${ }^{1} \mathrm{P}$ Naluyima, ${ }^{4} \mathrm{D}$ B Meya, ${ }^{5} \mathrm{~J} \mathrm{H}$ Rowe, 'F Cham, ${ }^{5} \mathrm{D}$ R Boulware. ${ }^{1}$ Makerere university walter reed project, Kampala, Uganda; ${ }^{2}$ Macalester college, St. Paul, MN, United States; ${ }^{3}$ Makerere university medical school, Kampala, Uganda; 4 Infectious diseases institute, Kampala, Uganda; ${ }^{5}$ University of Minnesota, Minneapolis, MN, United States

Background Cryptococcus meningitis immune reconstitution inflammatory syndrome (CM-IRIS) is a medical condition that complicates recovery from immunodeficiency as a result of anti retroviral therapy (ART) in patients living with Human Immunodeficiency Virus type 1 (HIV-1) in the sub Sahara Africa region. 
Methods This study investigated CD4 T cell immune activation based on intracellular IFN- $\gamma$ and Ki-67 expression, after ex-vivo cryptococcal antigen stimulation of whole blood samples taken from HIV-1 positive adult patients infected with or without cryptococcal meningitis, initiated on ART.

Results In the CM positive group at pre-ART visit; stimulation with C.neoformans crude cell wall (CW) induced a significant increase in CD4 IFN- $\gamma$ production $\left(p<0.05^{* * * *}\right)$, as compared to C.neoformans glucuronoxylomannan (GXM) polysaccharide antigen $\left(p<0.05^{*}\right)$, whilst C.neoformans mannoprotein (MP) stimulation failed to induce greater than baseline IFN- $\gamma$ expression. The effector memory $\mathrm{T}$ cell subset was the major contributor to the IFN- $\gamma$ elevation exhibited in CW stimulated samples. Interestingly, $T$ cell responses to CW were found to be significantly higher in the CM positive group compared to the CM negative group $\left(\mathrm{p}<0.05^{*}\right)$. Furthermore, stimulation with CW and GXM exhibited higher frequency of terminally differentiated effector memory $\mathrm{T}$ cells (TDEMS) compared to either negative control or MP stimulation. Conclusion Immune activation of CD4 T cells can be achieved by C.neoformans $\mathrm{CW}$ rather than purified MP antigen, by inducing the effector memory subset to produce IFN- $\gamma$.

\section{P5.075 BIOPLEX ${ }^{\circledR} 2200$ HIV AG-AB: AN AUTOMATED SCREENING METHOD PROVIDING DISCREET DETECTION OF HIV-1 P24, HIV-1 ANTIBODY, AND HIV-2 ANTIBODY}

doi:10.1136/sextrans-2013-051184.1119

W Link, R del Rosario, M Leos, A Carillo, D King, M Baumeister. Bio-Rad Laboratories, Benicia, CA, United States

Background Develop an automated HIV assay with $4^{\text {th }}$ generation sensitivity that can report antibody and antigen results individually, and distinguish HIV-1 from HIV-2 positives.

Methods The BioPlex $2200 \mathrm{HIV}$ Ag-Ab assay uses multiplex flow immunoassay to detect HIV-1 p24 antigen, HIV-1 antibody (Groups $\mathrm{M}$ and $\mathrm{O}$ ), and HIV-2 antibody in a single reaction vessel using a mixture of four populations of dyed microparticles. Each population is coated with a different HIV antigen or with p24 antibody. Results for each marker can be reported individually, and antibodyreactive specimens can be typed as HIV-1 or HIV-2. Specimens with similar levels of HIV-1 and HIV-2 antibody reactivity are reported as reactive but undifferentiated.

Results of in-house testing: To assess specificity, 5239 samples of unknown risk were tested resulting in specificity of $99.83 \%$. To assess sensitivity, known positive specimens (209 HIV-1 Group M, 21 HIV-1 Group O, and 177 HIV-2) were tested and all were reactive. All HIV-1 samples (209 Group M and 21 Group O) were correctly identified as HIV-1, and 153 of 177 HIV-2 samples were correctly identified as HIV-2. Of 24 that were undifferentiated, 18 could not be typed by Orgenics Immunocomb ${ }^{\circledast}$. Among 26 commercial seroconversion panels, BioPlex 2200 detected HIV-1 infection one donation sooner than Abbott Architect Combo HIV (4 $4^{\text {th }}$ generation) in three panels. In one panel, BioPlex 2200 missed one donation positive by Architect. Both tests gave equivalent results for 22 of the 26 panels.

Conclusion The BioPlex 2200 HIV Ag-Ab assay, which is currently in development, is highly sensitive and specific, and can also provide detailed screening results that will assist in identifying specimens from primary infection and HIV-2 positives and guide selection of of supplemental testing.

\section{P5.076 DEEP MYCOSES IN PATIENTS INFECTED WITH HUMAN IMMUNODEFICIENCY VIRUS (HIV) IN MONTEVIDEO, URUGUAY}

doi:10.1136/sextrans-2013-051184.1120

M Carbia, A Otero, B Ana Laura, B Raquel. Clinical Hospital. Medicine School, Montevideo, Uruguay
Background Deep fungal infections have been a major cause of morbidity and mortality in HIV-infected host, gaining importance due to their severity and poor prognosis. Because instituted antiretroviral therapy in patients infected with HIV in the last decade, it is expected that the profile of the deep mycoses in our country has changed, despite the increase in cases of HIV infection registered. The objective of this study was to report the frequency of deep mycosis in a population of HIV-positive patients in Montevideo, Uruguay.

Method The clinical fungal isolates obtained from biological samples, in HIV positive patients, processed in Section Mycology, Laboratory of Pathology University Hospital, between 2008 and 2012 The diagnosis of deep mycoses, was established by conventional mycological study (direct examination and culture). This was complemented by detection of antigen of Cryptococcus spp. in CSF samples, and direct immunofluorescence for Pneumocystis jirovecii in bronchoalveolar-lavage specimens.

Results Of 479 studies in HIV-positive patients, 248 were of cerebrospinal fluid, 83-bronchioloalveolar washes, 42 biopsies, 36 blood, 24 bone marrow, skin 20, and 26 belonged to other locations. 89 samples were found positive, of which 76 were from Cryptococcus spp.; 6 to Histoplasma capsulatum, Pneumocystis jirovecii 6 and 1 to Candida albicans isolated from the peritoneal fluid. Discussion: The frequency of deep mycoses was $18.6 \%$ of the samples studied. Cryptococcosis and meningo-encephalic presentation was the most frequent (75\% of samples positive for Cryptococcus spp.), histoplasmosis, and pneumocystosis represented $7 \%$ each of the total positive samples. The deep mycoses remains so similiar to previous periods in our country, persisting as a real problem in this population. The distribution of etiologic agents remained significantly unchanged as Cryptococcus spp. main exponent, but still keeps a significant decrease in the frequency and Pneumocystis jirovecii, Histoplasma capsulatum, and the absence of cases of aspergillosis.

\section{P5.077 NUCLEIC ACID AMPLIFICATION TEST (NAAT) DIAGNOSTICS COMBINED WITH DELAYED NEISSERIA GONORRHOEAE CULTIVATION OF NAAT POSITIVE SAMPLES USING THE ESWAB ${ }^{\text {TM }}$ SYSTEM - THE SOLUTION FOR FUTURE GONOCOCCAL ANTIMICROBIAL SUSCEPTIBILITY SURVEILLANCE?}

doi:10.1136/sextrans-2013-051184.1121

${ }^{1,2}$ C M Wind, ${ }^{1,2.3} \mathrm{H} \mathrm{J} \mathrm{C}$ de Vries, ${ }^{4} \mathrm{M}$ Unemo, ${ }^{5,6} \mathrm{~A}$ P van Dam. ${ }^{1}$ STI Outpatient clinic, Cluster Infectious Diseases, Municipal Health Service Amsterdam, Amsterdam, The Netherlands, '2Dept. of Dermatology, Academic Medical Center, University of Amsterdam, Amsterdam, The Netherlands; ${ }^{3}$ Centre for Infectious Disease Control, National Institute for Public Health and the Environment, Bilthoven, The Netherlands; ${ }^{4}$ WHO Collaborating Centre for Gonorrhoea \& Other STIS, National Reference Laboratory for Pathogenic Neisseria, Dept. of Laboratory Medicine, Microbiology, Örebro University Hospital, Örebro, Sweden; ${ }^{5}$ Public Health Laboratory, Cluster Infectious Diseases, Municipal Health Service Amsterdam, Amsterdam, The Netherlands; ${ }^{6}$ Dept. of Medical Microbiology, Onze Lieve Vrouwe Gasthuis general hospital, Amsterdam, The Netherlands

Background Antimicrobial resistance (AMR) in Neisseria gonorrhoeae $(\mathrm{Ng})$ is a major public health problem worldwide. Nucleic acid amplification tests (NAATs) have rapidly replaced cultivation for detection of $\mathrm{Ng}$. We have evaluated the ESwab system for NAAT diagnostics combined with delayed $\mathrm{Ng}$ cultivation of NAAT positive samples for gonococcal AMR surveillance.

Methods Based on clinical indications, a urethral, cervical or anal swab was collected from patients with purulent discharge. Gold standard for diagnosis was the APTIMA Combo 2 assay (GenProbe). In another study, swabs from urine (UR) and urine sediment (US) were collected if Gram-negative diplococci were observed in direct smears. Flocked swabs were stored in ESwab Liquid Amies (Copan) at room temperature (RT) and $4^{\circ} \mathrm{C}$ and cultured after 1, 24 and 48 hours. 